\title{
Circulating level of Angiopoietin-2 is associated with acute kidney injury in coronavirus disease 2019 (COVID-19)
}

\author{
Brandon Michael Henry ${ }^{1}$ ( ) Maria Helena Santos de Oliveira ${ }^{2} \cdot$ Isaac Cheruiyot ${ }^{3}$. Justin L. Benoit ${ }^{4}$. \\ David S. Cooper ${ }^{1,5}$. Giuseppe Lippi ${ }^{6}$. Timothy D. Le Cras ${ }^{5,7} \cdot$ Stefanie W. Benoit ${ }^{5,8}$
}

Received: 9 January 2021 / Accepted: 8 March 2021 / Published online: 23 March 2021

(c) The Author(s), under exclusive licence to Springer Nature B.V. 2021

\section{To the editor,}

Emerging evidence suggests that endothelial dysfunction plays a central role in the pathophysiology of coronavirus disease 2019 (COVID-19). Recent post-mortem studies have documented extensive endothelial damage and inflammatory infiltrates in pulmonary and extra-pulmonary capillary beds of COVID-19 patients [1, 2]. This results in loss of endothelial integrity, activation of pro-coagulant pathways, disruption of the alveolar-capillary barrier, and vascular hyperpermeability [2]. Endothelial damage is a common denominator of thrombosis (micro- and macrovascular), acute respiratory distress syndrome (ARDS), acute kidney injury (AKI), and multiorgan failure (MOF), which are major drivers of morbidity and mortality in COVID-19 patients [3]. AKI is a common feature of COVID-19, impacting nearly half of all hospitalized patients, and is associated with high mortality, especially among those requiring renal replacement therapy [4-6]. We have recently shown that AKI may be driven in

Brandon Michael Henry

brandon.henry@cchmc.org

1 The Heart Institute, Cincinnati Children's Hospital Medical Center, 3333 Burnet Ave, Cincinnati, OH 45229, USA

2 Department of Statistics, Federal University of Parana, Curitiba, Brazil

3 School of Medicine, University of Nairobi, Nairobi, Kenya

4 Department of Emergency Medicine, University of Cincinnati, Cincinnati, OH, USA

5 Department of Pediatrics, University of Cincinnati College of Medicine, Cincinnati, OH, USA

6 Section of Clinical Biochemistry, University of Verona, Verona, Italy

7 Division of Pulmonary Biology, Cincinnati Children's Hospital Medical Center, Cincinnati, OH, USA

8 Division of Nephrology and Hypertension, Cincinnati Children's Hospital Medical Center, Cincinnati, OH, USA
COVID-19 by a secondary thrombotic microangiopathy (TMA) phenomenon, as evidenced by low ADAMTS13 activity to von Willebrand factor (VWF:Ag) ratio [7]. However, the mechanism by which AKI occurs in COVID-19 has yet to be fully elucidated.

With the high frequency of AKI and thromboses in patients with COVID-19, biomarkers of endothelial damage/activation-related biomarkers have become of interest. Angiopoietin-1 (Ang-1) is an angiogenic growth factor that promotes vessel maturation and survival by activation of the Tie2 receptor (Tie2) on endothelial cells [8]. Ang-1 is expressed by pericytes and vascular smooth muscle cells and can stabilize endothelial functions by reducing inflammation and apoptosis of endothelial cells [9]. On the contrary, Angiopoietin-2 (Ang-2) enhances endothelial inflammation and hyperpermeability as it can act as an antagonist to Ang-1 and Tie2 signaling [9, 10]. We hypothesized that elevated Ang-2 would be associated with an increased risk for developing severe COVID-19-related AKI during the course of infection.

In this prospective observational study, adults ( $\geq 18$ years old) presenting to the University of Cincinnati Medical Center Emergency Department (ED) with respiratory symptoms at triage suggestive of COVID-19 and with positive reverse transcription-polymerase chain reaction (RT-PCR) test for COVID-19 via nasopharyngeal swab were enrolled. This study was approved by the University of Cincinnati institutional review board (IRB) and performed under a waiver of informed consent. Blood samples were collected via routine draws for clinical indications in the ED. Circulating levels of Ang-1 and Ang-2 were determined in EDTA plasma using an enzyme-linked immunosorbent assay following the manufacturer's instructions (R\&D Systems, Minneapolis, MN, USA) using a DS2 ELISA processing system (Dynex Technologies, Inc, Chantilly, Virginia, USA). Serum creatinine was measured using a kinetic alkaline picrate (modified Jaffe) method using either a Beckman Coulter 
AU480 Chemistry Analyzer (Brea, California, USA) or a Beckman Coulter AU5822 Chemistry Analyzer (Brea, California, USA). Patients were monitored through hospitalization until discharge/death if admitted from the ED or for 30 days if discharged from the ED. The primary outcome of interest was the development of severe AKI, defined as Kidney Disease: Improving Global Outcomes (KDIGO) Stage $2+3$ according to serum creatinine ( $\mathrm{SCr}$ ) criteria [11]. The secondary outcome was the need for renal replacement therapy (RRT). Ang-2 levels were correlated with white blood cell count (WBC), C-reactive protein (CRP), interleukin (IL) $6,8,10$, tumor necrosis factor-alpha (TNF- $\alpha$ ), plasminogen, fibrinogen, D-Dimer, ADAMTS13 activity, VWF:ag, myoglobin, plasma neutrophil gelatinase-associated lipocalin (NGAL), and serum cystatin C.

Analysis of data was carried out using R software (version 4.0.2, R Foundation for Statistical Computing, Vienna, Austria). Categorical data were reported as frequencies (\%), while continuous data were reported as the median and interquartile range (IQR). Comparison of baseline Ang-1 and Ang-2 levels, as well as other laboratory values between COVID-19 patients with and without severe AKI, was carried out using the Mann-Whitney U-test. Proportions were compared between groups using Fisher's exact test. Logistic regression analysis was performed to estimate the effect of changes in Ang-1 and Ang-2 levels when adjusted for the presence of comorbidities, and variable selection was performed using the stepwise algorithm.

A total of 51 COVID-19 patients were included. The median age was 50.5 (IQR: 39.3-66.0) years, and 57.7\% were males. Their comorbidities are shown in Table 1. A total of $12(23.5 \%)$ COVID-19 patients developed severe AKI, 8 (66.6\%) needing RRT, and 3 (25.0\%) died. No significant differences were observed in Ang-1 levels (2904.1 [IQR: 737.5-5111.] vs. 2670.7 [IQR: 1321.6-4711.] $\mathrm{pg} / \mathrm{mL} ; p=0.916)$ or Ang-2/Ang-1 ratio (0.45 [IQR: $0.07-1.08$ ] vs. 1.15 [IQR: $0.53-2.47] \mathrm{pg} / \mathrm{mL} ; p=0.201$ ) in those who developed severe AKI versus those who did not. Nonetheless, Ang-2 levels were found to be significantly higher in those who developed severe AKI (4715.7 [IQR: 2768.8-17,919.1] vs. 2462.4 [IQR: 1699.0-3641.8] pg/mL; $p=0.047$ ) (Fig. 1a). Moreover, Ang-2 level was the highest in those who required RRT (13,372.7 [IQR: 3604.4-20000] vs. 2556.1 [IQR: 1699-3235] pg/mL; $p=0.037$ ) (Fig. 1b).

Table 1 Baseline demographics of the Cincinnati emergency department COVID-19 cohort

\begin{tabular}{|c|c|c|c|c|}
\hline \multirow[t]{2}{*}{ Variable } & \multirow[t]{2}{*}{ All patients $(n=51)$} & \multicolumn{2}{|l|}{ KDIGO AKI stage } & \multirow[t]{2}{*}{$p$-value } \\
\hline & & $0+1$ & $2+3$ & \\
\hline Age (years): median (IQR) & $50.5(41-66)$ & $47(37.5-64.0)$ & $66(56.5-70.2)$ & 0.005 \\
\hline Sex (male): n (\%) & 30 & $23(76.7 \%)$ & $7(23.3 \%)$ & 1.000 \\
\hline BMI: median (IQR) & $28.5(24.8-33.5)$ & $29.5(25.8-34.5)$ & $24.5(21.6-27.5)$ & 0.018 \\
\hline \multicolumn{5}{|l|}{ Race: $\mathrm{n}(\%)$} \\
\hline Black & 21 & $12(57.1 \%)$ & $9(42.9 \%)$ & \multirow[t]{4}{*}{0.036} \\
\hline Hispanic & 18 & $17(94.4 \%)$ & $1(5.6 \%)$ & \\
\hline White & 9 & $7(77.8 \%)$ & $2(22.2 \%)$ & \\
\hline Other & 3 & $3(100 \%)$ & $0(0 \%)$ & \\
\hline \multicolumn{5}{|l|}{ Comorbidities: n (\%) } \\
\hline Coronary artery disease & 8 & $3(37.5 \%)$ & $5(62.5 \%)$ & 0.012 \\
\hline Heart failure & 9 & $3(33.3 \%)$ & $6(66.7 \%)$ & 0.003 \\
\hline Hypertension & 26 & $15(57.7 \%)$ & $11(42.3 \%)$ & 0.002 \\
\hline Hyperlipidemia & 15 & $11(73.3 \%)$ & $4(26.7 \%)$ & 0.730 \\
\hline Diabetes & 21 & $15(71.4 \%)$ & $6(28.6 \%)$ & 0.738 \\
\hline Chronic obstructive pulmonary disease & 8 & $4(50 \%)$ & $4(50 \%)$ & 0.076 \\
\hline Asthma & 8 & $6(75 \%)$ & $2(25 \%)$ & 1.000 \\
\hline Chronic kidney disease & 6 & $1(16.7 \%)$ & $5(83.3 \%)$ & 0.002 \\
\hline Chronic liver disease & 7 & $3(42.9 \%)$ & $4(57.1 \%)$ & 0.044 \\
\hline Cerebrovascular disease & 1 & $0(0 \%)$ & $1(100 \%)$ & 0.375 \\
\hline Cancer & 4 & $1(25 \%)$ & $3(75 \%)$ & 0.036 \\
\hline Acquired immunodeficiency (HIV, transplant) & 3 & $2(66.7 \%)$ & $1(33.3 \%)$ & 1.000 \\
\hline Autoimmune disease & 2 & $2(100 \%)$ & $0(0 \%)$ & 1.000 \\
\hline
\end{tabular}

* BMI Body Mass Index, KDIGO Kidney Disease: Improving Global Outcomes, AKI Acute Kidney Injury $p<0.05$ 
A

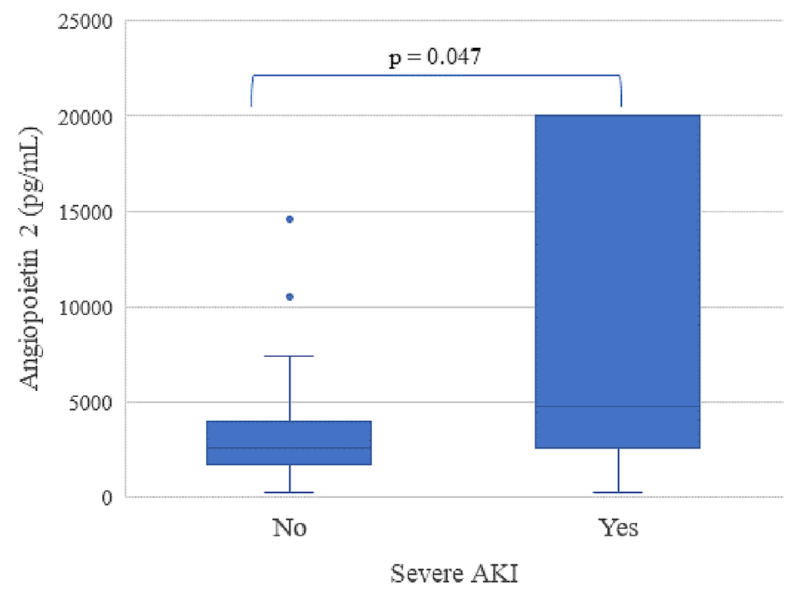

B

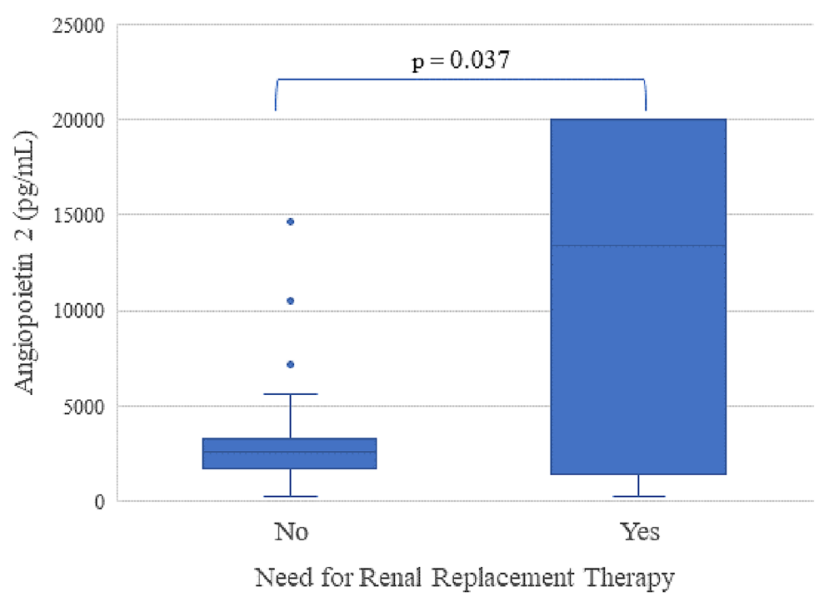

Fig. 1 Angiopoietin-2 levels in patients developing severe AKI (a) and in patients requiring renal replacement therapy (RRT) (b)

Ang-2 was found to be positively correlated with WBC $(r=0.596 ; p<0.001)$, IL-6 $(r=0.280 ; p=0.049)$, TNF- $\alpha$ ( $r=0.316 ; p=0.024)$, fibrinogen $(r=0.405 ; p=0.009)$, D-dimer $(r=0.552 ; p=0.008)$, cystatin C $(r=0.345$, $p=0.019)$, NGAL $(r=0.431, p=0.002)$, and negatively correlated with plasminogen $(r=-0.370 ; p=0.007)$ and ADAMTS13 $(r=-0.302 ; p=0.031)$. No correlation was observed for IL-10 ( $p=0.794)$, CRP $(p=0.11)$, or VWF:ag $(p=0.427)$.

In multivariate logistic regression, both pre-existing chronic kidney disease and hypertension were significantly associated with increased odds of severe AKI, with adjusted odds ratios (ORs) of 31.8 (95\%CI 1.18-854.88) and 22.0 (95\%CI 1.15-420.32), respectively. An increase in 1000 pg/ $\mathrm{mL}$ of Ang-2 was associated with a $39 \%$ increase in odds of severe AKI (OR 1.39 [95\%CI 1.05-1.86]). Full results are presented in Supplemental Table 1.

In this prospective study, we observed that Ang-2 levels measured at ED presentation are significantly increased in patients at risk of developing severe AKI. Moreover, we observed that elevated Ang-2 is an independent predictor of severe AKI and RRT. Our findings are in agreement with Smadja et al. [12], who reported significantly higher levels of Ang-2 in intensive care unit-admitted COVID-19 patients. They observed that patients with Ang-2 levels greater than $5000 \mathrm{pg} / \mathrm{mL}$ had ninefold higher odds of ICU admission. Our findings are also in agreement with Araujo et al. [13] who observed that elevated Ang-2 levels were significantly associated with increased odds of severe AKI and need for RRT in ICU-admitted non-COVID-19 acute respiratory distress syndrome (ARDS) patients.

Overall, our results are consistent with a picture of endothelial injury and a thrombotic microangiopathy phenomenon in COVID-19-associated AKI, further supported by the negative correlation with ADAMTS13 activity and positive correlations with fibrinogen and D-dimer. These results are consistent with elevations of Ang-2 observed in other forms of TMA [14-16]. Ang-2 was also correlated with several pro-inflammatory biomarkers, consistent with a hyperinflammatory response that can produce endothelial injury. Endothelium activation can lead to the release of Ang-2 from Weibel-Palade (WP) bodies [17]. Interestingly, however, we did not observe significant correlation between Ang-2 and VWF:ag. Philippe et al. [18] reported observing two distinct biomarker profiles, with VWF:ag increased in accordance with disease severity, while Ang-2 was elevated only in the critically ill. Taken together, this suggests that endothelial VWF secretion in COVID-19 may in part occur via pathways different than that of Ang-2. Indeed, while VWF is also secreted via WP bodies in the basal and regulated secretory pathways, the endothelium may also directly secrete VWF via a constitutive secretory pathway using small anterograde carriers [19]. Moreover, COVID-19 is associated with platelet hyperactivity, which occurs via multiple mechanisms, including spike protein binding to platelet angiotensin-converting enzyme 2 (ACE2) receptors, resulting in platelet activation and alpha granule release, which contains VWF in high molecular weight forms [20].

Ang-2 inhibits the protective anti-inflammatory Ang-1/ Tie2 signaling cascade [17]. The Tie 2 receptor is a central regulator in protecting the vasculature against thrombus formation in the setting of systemic inflammation, such as that seen in sepsis [21]. In a pilot study of critically ill patients with TMA and anti-glomerular basement membrane disease, plasma exchange was shown to be an effective method to remove excess circulating Ang-2, returning to almost normal values with $\leq 4$ treatments [17]. As such, the investigation of the pathophysiologic role of Ang-2 in COVID-19 should be prioritized as targeting Ang-2 via plasma exchange or other inhibitory approaches are potential therapies in patients with 
severe COVID-19. Future longitudinal studies are needed to fully elucidate the role of Ang-2 in COVID-19 endothelial dysfunction and multiorgan injury and the specificity of Ang-2 for COVID-19 AKI.

Supplementary Information The online version contains supplementary material available at https://doi.org/10.1007/s10456-021-09782-w.

Funding This study was funded by the University of Cincinnati College of Medicine Special Coronavirus (COVID-19) Research Pilot Grant Program and the Lymphatic Malformation Institute.

\section{Declarations}

Conflict of interest The authors do not have any conflicts of interest concerning this publication.

\section{References}

1. Bradley BT, Maioli H, Johnston R et al (2020) Histopathology and ultrastructural findings of fatal COVID-19 infections in Washington State: a case series. The Lancet 396:320-332

2. Varga Z, Flammer AJ, Steiger P et al (2020) Endothelial cell infection and endotheliitis in COVID-19. Lancet 395:1417-1418

3. Pons S, Fodil S, Azoulay E et al (2020) The vascular endothelium: the cornerstone of organ dysfunction in severe SARS-CoV-2 infection. Crit Care 24:353

4. Gupta S, Coca SG, Chan L, et al. (2020) AKI Treated with Renal Replacement Therapy in Critically Ill Patients with COVID-19. JASN [Internet]. [cited 2020 Nov 11]; Available from: https://jasn. asnjournals.org/content/early/2020/10/15/ASN.2020060897.

5. Chan L, Chaudhary K, Saha A, et al. (2020) AKI in Hospitalized Patients with COVID-19. JASN [Internet]. [cited 2020 Nov 11]; Available from: https://jasn.asnjournals.org/content/early/2020/ 09/02/ASN.2020050615.

6. Cheruiyot I, Henry B, Lippi G, et al. Acute Kidney Injury is Associated with Worse Prognosis In COVID-19 Patients: A Systematic Review and Meta-analysis. 1. 2020;91:ahead of print-ahead of print.

7. Henry BM, Benoit SW, de Oliveira MHS et al (2020) ADAMTS13 activity to von Willebrand factor antigen ratio predicts acute kidney injury in patients with COVID-19: Evidence of SARS-CoV-2 induced secondary thrombotic microangiopathy. Int J Lab Hematol. https://doi.org/10.1111/ijlh.13415

8. Yancopoulos GD, Davis S, Gale NW et al (2000) Vascular-specific growth factors and blood vessel formation. Nature 407:242-248
9. Neuhauß A-K, Gutbier B, Friedemann T, et al. Angiopoietins: Possible biomarkers in severe pneumonia? European Respiratory Journal [Internet]. 2012 [cited 2020 Nov 5];40. Available from: https://erj.ersjournals.com/content/40/Suppl_56/P830.

10. Thurston G, Daly C (2012) The complex role of angiopoietin-2 in the angiopoietin-tie signaling pathway. Cold Spring Harb Perspect Med 2:a006550

11. KDIGO AKI Working Group (2012) KDIGO clinical practice guideline for acute kidney injury. Kidney International Suppl 2:1

12. Smadja DM, Guerin CL, Chocron R et al (2020) Angiopoietin-2 as a marker of endothelial activation is a good predictor factor for intensive care unit admission of COVID-19 patients. Angiogenesis 23(4):611-620

13. Araújo CB, de Oliveira Neves FM, de Freitas DF et al (2019) Angiopoietin-2 as a predictor of acute kidney injury in critically ill patients and association with ARDS. Respirology 24:345-351

14. Lukasz A, Beneke J, Thamm K, et al. Involvement of Angiopoietin-2 and Tie2 Receptor Phosphorylation in STEC-HUS Mediated by Escherichia coli O104:H4 [Internet]. Mediators of Inflammation. Hindawi; 2015 [cited 2020 Dec 29]. p. e670248. Available from: https://www.hindawi.com/journals/mi/2015/670248/.

15. Ueda N, Chihara D, Kohno A et al (2014) Predictive Value of Circulating Angiopoietin-2 for Endothelial Damage-Related Complications in Allogeneic Hematopoietic Stem Cell Transplantation. Biol Blood Marrow Transplant 20:1335-1340

16. Shimizu M, Inoue N, Kuroda M et al (2017) Angiopoietin-1 and -2 as markers for disease severity in hemolytic uremic syndrome induced by enterohemorrhagic Escherichia coli. Clin Exp Nephrol 21:76-82

17. Lovric S, Lukasz A, Hafer C et al (2010) Removal of elevated circulating angiopoietin-2 by plasma exchange-a pilot study in critically ill patients with thrombotic microangiopathy and anti-glomerular basement membrane disease. Thromb Haemost 104:1038-1043

18. Philippe A, Chocron R, Gendron N et al (2021) Circulating Von Willebrand factor and high molecular weight multimers as markers of endothelial injury predict COVID-19 in-hospital mortality. Angiogenesis. https://doi.org/10.1007/s10456-020-09762-6

19. Lopes da Silva M, Cutler DF (2016) von Willebrand factor multimerization and the polarity of secretory pathways in endothelial cells. Blood 128:277-285

20. Zhang S, Liu Y, Wang X et al (2020) SARS-CoV-2 binds platelet ACE2 to enhance thrombosis in COVID-19. J Hematol Oncol 13:120

21 Higgins SJ, De Ceunynck K, Kellum JA et al (2018) Tie2 protects the vasculature against thrombus formation in systemic inflammation. J Clin Invest. 128:1471-1484

Publisher's Note Springer Nature remains neutral with regard to jurisdictional claims in published maps and institutional affiliations. 